\title{
Protecting your Future from Diabetes
}

\author{
Sachin Chaudhary* \\ Department of Medicinal Chemistry, University of Sharjah, United Arab Emirates
}

*Corresponding author: Sachin Chaudhary, Department of Medicinal Chemistry, College of Pharmacy, University of Sharjah, P.B. 27272, United Arab Emirates

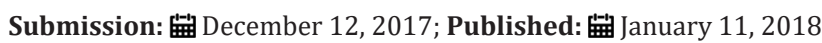

\section{Abstract}

Diabetes is disabling, deadly and on the rise. In fact, diabetes is at epidemic proportions around the globe as 415 million people globally have diabetes, if nothing is done, it is estimated to rise to 642 million in 2040. Diabetes can shorten an individual's life by as much as 15 years.

Keywords: Diabetes; Epidemic; Life

\section{Introduction}

There are three main types of Diabetes. Type-I (Juvenile-onset diabetes), it is usually caused by an autoimmune reaction where the body's defense system attacks the cells that produce insulin. Type-II diabetes (Non-insulin dependent diabetes). It accounts for at least $90 \%$ of all cases of diabetes. It is characterized by Insulin resistance and relative insulin deficiency, either or both of which may be present at the time diabetes is diagnosed [1]. Gestational diabetes (GDM) is a form of diabetes consisting of high blood glucose levels during pregnancy. It develops in one in 25 pregnancies worldwide and is associated with complications to both mother and baby. GDM usually disappears after pregnancy but women with GDM and their children are at an increased risk of developing type-II diabetes later in life. Approximately half of women with a history of GDM go on to develop type-II diabetes within five to ten years after delivery [2,3].

\section{Discussion}

A complex relationship between insulin, glucose, your liver and other hormones ensures that your blood sugar stays within set limits. Here is how it works:

i. When you eat, your body breaks down carbohydrates from foods into various sugar molecules. One of these is glucose, which is absorbed directly into your bloodstream, to be used or stored.

ii. When you do not eat, your liver (and kidney) produce sugar for fuel.

iii. Insulin causes glucose to be absorbed into your cells, where it can be used for energy.

iv. The pancreas produces insulin and regulates how much is released into your body.

v. If you do not eat for a long period, the pancreas limits the release of insulin. vi. After a meal or a snack, the pancreas sends extra insulin into your bloodstream.

vii. If you have more glucose than your cells need, your body stores the excess in your liver as glycogen until it is needed.

If all goes well, your body maintains "normal" blood sugar levels. If there is not enough insulin or your body cannot use it properly, glucose accumulates in the blood instead of going into cells. When blood glucose levels are chronically too high, you have diabetes.

\section{Diabetes complications}

People with diabetes have an increased risk of developing numerous health problems like:

Cardiovascular disease: Affects the heart and blood vessels and may cause fatal complications such as coronary artery disease (leading to heart attack) and stroke, high blood pressure, high cholesterol and other risk factors contribute to increasing the risk of cardiovascular complications.

Kidney disease (diabetic nephropathy): Caused by damage to small blood vessels in the kidneys leading to dysfunctioning. Kidney disease is much more common in people with diabetes than in those without diabetes.

Nerve disease (diabetic neuropathy): Diabetes can cause damage to the nerves throughout the body when blood glucose and blood pressure are too high. This can lead to problems with digestion, erectile dysfunction, and many other functions. Among the most commonly affected areas are the extremities, in particular the feet. Nerve damage in these areas is called peripheral neuropathy, and can lead to pain, tingling, and loss of feeling. Loss of feeling is particularly important because it can allow injuries to go unnoticed, leading to serious infections and possible amputations. 
Eye disease (diabetic retinopathy): Most people with diabetes will develop some form of eye disease (retinopathy) causing reduced vision or blindness. Consistently high levels of blood glucose, together with high blood pressure and high cholesterol, are the main causes of retinopathy.

Diabetes prevention: At present, type 1 diabetes cannot be prevented. The environmental triggers that are thought to generate the process that results in the destruction of the body's insulinproducing cells are still under investigation. While there are a number of factors that influence the development of type-II diabetes, it is evident that the most influential are lifestyle behaviors including consumption of processed foods, foods with a high fat content, sugar-sweetened beverages and highly refined carbohydrates. At the same time, modern lifestyles are characterized by physical inactivity and long sedentary periods. Together, these behaviors are associated with an increased risk of being overweight or obese and the development of type-II diabetes. A number of prevention programmes have shown that modifying such behaviors, by eating healthier foods and increasing physical activity, can greatly reduce the risk of developing type 2 diabetes. In order to halt the increase of type 2 diabetes, whole populations must change their lifestyle behaviors by modifying diet and increasing physical activity levels.

\section{Recommendations for a healthy diet for the general population}

i. Choosing water, coffee or tea instead of fruit juice, soda, or other sugar sweetened beverages.

ii. Eating at least three servings of vegetable every day, including green leafy vegetables.

iii. Eating up to three servings of fresh fruit every day.

iv. Choosing nuts, a piece of fresh fruit, or unsweetened yoghurt for a snack. v. Limiting alcohol intake to a maximum of two standard drinks per day.

vi. Choosing lean cuts of white meat, poultry or seafood instead of red or processed meat.

vii. Choosing peanut butter instead of chocolate spread or jam.

viii. Choosing whole-grain bread, rice, or pasta instead of white bread, rice, or pasta.

ix. Choosing unsaturated fats (olive oil, canola oil, corn oil, or sunflower oil) instead of saturated fats (butter, ghee, animal fat, coconut oil or palm oil.

A particular threat in terms of the associated risk of developing type 2 diabetes is the consumption of high sugar foods, particularly sugar-sweetened beverages.

\section{Conclusion}

However, there is no cure for Type-II Diabetes, it can be managed and the onset of complications delayed. Better yet, TypeII Diabetes itself can often be prevented when people make good lifestyle choices and make them early enough in their lives.

\section{References}

1. Chaudhary S, Semwal A, Kumar H, Verma HC (2016) In-vivo study for anti-hyperglycemic potential of aqueous extract of Basil seeds (Ocimumbasilicum Linn) and its influence on biochemical parameters, serum electrolytes and hematological indices. Biomed Pharmacother 84: 2008-2013.

2. Alfadhli EM (2015) Gestational diabetes mellitus. Saudi Med J 36(4): 399-409.

3. Agarwal MM (2015) Gestational diabetes mellitus: An update on the current international diagnostic criteria. World J Diabetes 6(6): 782791. 